\title{
AC-STEM Studies of Phase Transformation and Evolution of Li-Rich Layered Cathode Materials Induced by Battery Charge-Discharge Cycling and by Electron-beam Irradiation
}

\author{
Ping Lu ${ }^{1}$, Pengfei Yan ${ }^{2}$, Eric Romero ${ }^{1}$, Erik David Spoerke ${ }^{1}$, Ji-Guang Zhang ${ }^{3}$, and Chong-Min \\ Wang $^{2}$ \\ ${ }^{1}$ Sandia National Laboratories, P O Box 5800, Albuquerque, NM 87185, USA \\ ${ }^{2}$ Environmental Molecular Sciences Laboratory, Pacific Northwest National Laboratory, 902 \\ Battelle Boulevard, Richland, WA 99352, USA \\ ${ }^{3}$ Energy and Environmental Directorate, Pacific Northwest National Laboratory, 902 Battelle \\ Boulevard, Richland, WA 99352, USA
}

Structural and chemical imaging of defects and crystal structures at the atomic-scale has become possible by recent technical advances in aberration-corrected scanning transmission electron microscopy (AC-STEM) and x-ray detector technology [1]. AC-STEM imaging using high-angle annular dark-field (HAADF) detector and chemical mapping using energy-dispersive $\mathrm{x}$-ray spectroscopy (EDS) have been used in combination to resolve interfaces, defects and crystalline structures unambiguously [2]. The AC-STEM capability has also been recently used to study layered lithium and manganese-based materials, such as the lithium-rich, manganeserich (LMR) oxides (e.g., $\mathrm{Li}\left[\mathrm{Li}_{\mathrm{x}} \mathrm{Mn}_{\mathrm{y}} \mathrm{TM}_{1-\mathrm{x}-\mathrm{y}}\right] \mathrm{O}_{2}(\mathrm{TM}=$ transition metal, e.g., Ni, Co or Fe)), which are of great interest as cathode materials for secondary (rechargeable) lithium ion batteries [3]. These studies have largely attributed the loss of capacity and voltage in the layered oxides upon the charge-discharging cycling to the development of surface reconstructed layers (SRLs) on cathode particle surfaces. On the other hand, lithium-containing oxides are known to be prone to radiation damage by high-energy electron beams. In this study, we report a comparative ACSTEM study on the structural evolution of the surface layer on LMR $\mathrm{Li}\left[\mathrm{Li}_{0.2} \mathrm{Ni}_{0.2} \mathrm{Mn}_{0.6}\right] \mathrm{O}_{2}$ (LNMO) nanoparticles (NPs) induced by high-energy electron-beam irradiation and by chargedischarge cycling [4].

Unless extreme caution in controlling electron exposure was taken during STEM imaging, the surfaces of LNMO NPs were always found to have the SRLs which resemble those reported due to charge-discharge cycling [3]. Fig.1 shows the phase transformation and evolution on the surfaces of LNMO due to electron-beam irradiation. The original state of the NPs without the SRL(Fig.1a) was quickly transformed to a thin, defect spinel SRL after exposed to a $200 \mathrm{kV}$ electron beam for as little as 30 seconds under normal high-resolution STEM imaging conditions (with an equivalent electron beam current-density of about $18 \mathrm{~A} / \mathrm{cm}^{2}$ ). Further electron irradiation produced a thicker layer of the spinel phase, ultimately creating a rock-salt layer at a higher electron exposure (Fig.1c). Fig.2 shows the surfaces of LNMO NPs after the electrochemical charge-discharge cycling. Both the defect spinel structure and the rock-salt structure are visible near the edge of the particle. Atomic-scale chemical mapping by EDS in STEM has also been used to study the electron-beam-induced SRL formation on LNMO. The result of the EDS study indicates the transformation is accomplished by migration of the transition metal ions to the Li sites without breaking down the lattice [4, 5].

The observations in this study enable a better understanding of the mechanism of forming the SRL and its structural evolution in the Li-rich layered oxides during the cycling, and also points to the special caution necessary when using TEM or STEM for the study of the lithium layered oxides and other similar oxides. 
References:

1. Von Harrach, H.S., Dona, P., Freitag, B., Soltau, H., Niculae, A. \& Rohde, M. (2009). Microsc. Microanal. 15 (suppl.2) 208-209.

2. P. Lu, J. Xiong, M. Van Benthem . \& Q.X. Jia App. Phys. Lett. 102, 173111 (2013); P. Lu, E. Romero, S. Lee, J. L. MacManus-Driscoll \& Q. X. Jia, Microsc. Microanal. 20, 17821792 (2014); P. Lu, L. Zhou, M.J. Kramer \& D. J. Smith, Sci. Rep. 4, 3945-3949 (2014).

3. A. Boulineau, L. Simonin, J. -F. Colin, J.-F.; C. Bourbon, \& S. Patoux, Nano Lett. 13, 3857 (2013); B. Xu, C.R. Fell, M. Chi, Y.S. Meng, Energ. Environ. Sci. 4, 2223 (2011); F. Lin, I.M. Markus, D. Nordlund, T.-C. Weng, M.D. Asta, H. L. Xin \& M.M. Doeff, Nat. Commun. 5, 3529 (2014)

4. P. Lu, P. F. Yan' E. Romero, E. D. Spoerk, J.-G. Zhang and C.-M. Wang, Chemistry of Materials, accepted. (2015).

5. Sandia National Laboratories is a multi-program laboratory managed and operated by Sandia Corporation, a wholly owned subsidiary of Lockheed Martin Corporation, for the US Department of Energy's National Nuclear Security Administration under contract DE-AC0494AL85000.
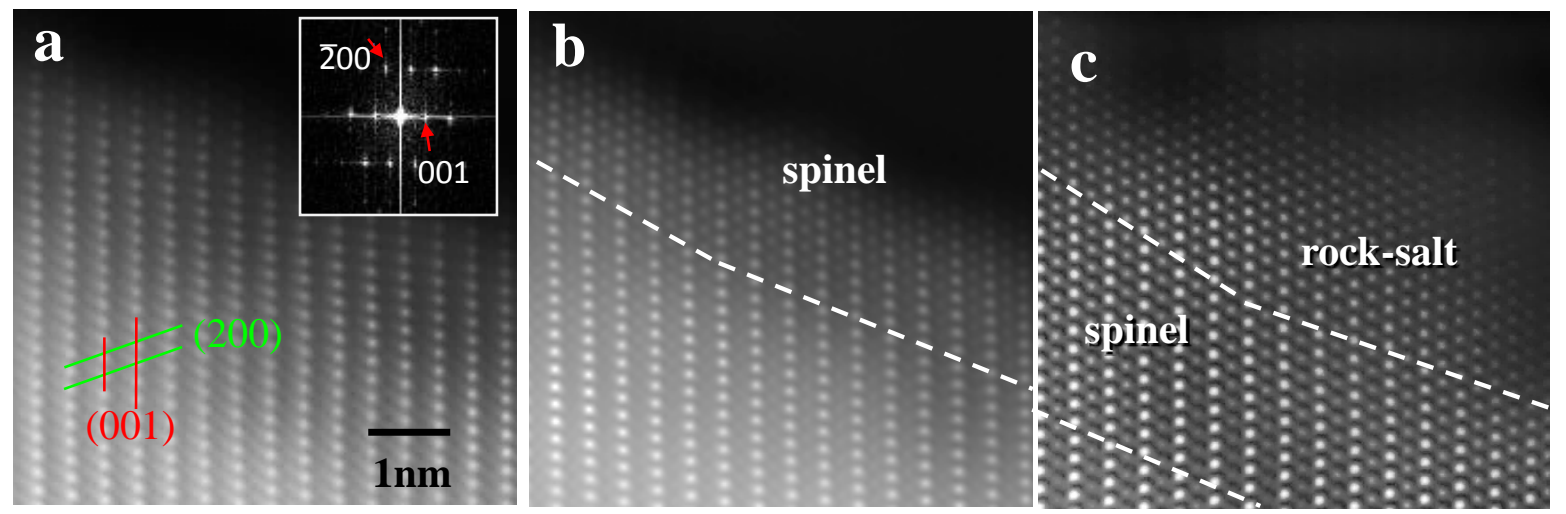

Fig.1. High-resolution STEM HAADF images showing the effect of electron-beam irradiation: (a) original state of LNMO NPs obtained without electron beam pre-exposure; (b) and (c) taken after electron-beam irradiation for 30 seconds and 200 seconds respectively under normal highresolution STEM viewing conditions (a current-density of about $18 \mathrm{~A} / \mathrm{cm}^{2}$, at dwell-time of $\sim 1$ $\mu$ s per pixel). Images are taken with the bulk in [010] direction (space group C2/m), as seen from FFT pattern in inset. The dash lines indicate the approximate positions separating the SRL from the bulk. Major lattice planes are marked for the bulk in (a).

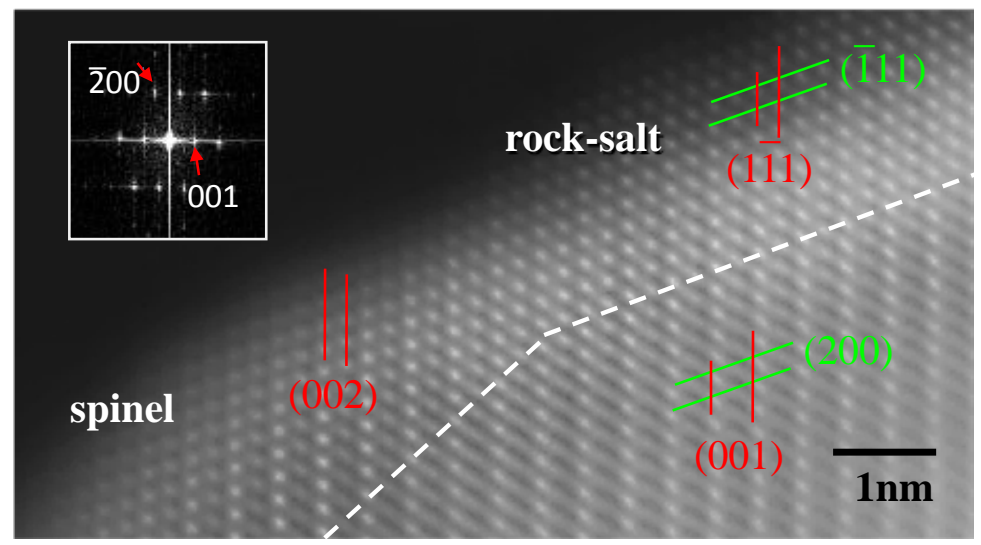

Fig.2. High-resolution STEM HAADF image showing the SRLs formed due to the electrochemical charge-discharge cycling. The dash lines indicate the approximate positions separating the SRL from the bulk. Major lattice planes are marked in the respective phases. Images are taken with the bulk in [010] direction (space group C2/m). 